\section{Luminescence Photophysics in Semiconductor Nanocrystals}

\author{
MANOJ NIRMAL AND LOUIS BRUS*
}

Chemistry Department, Columbia University, New York, New York 10027

Received April 20, 1998

\section{Introduction}

Sp3-hybridized semiconductors (including InP, GaAs, $\mathrm{CdSe}$, and $\mathrm{Si}$ ) are remarkable from the perspective of physical chemistry. A single electron created by HOMOLUMO promotion moves rapidly in response to an applied electric field, because there is little lattice distortion (i.e., small Franck-Condon factors) accompanying its creation. According to Marcus-Hush electron transfer theory, electron motion is resonant in the limit of vanishing reorganization energy. Franck-Condon factors are also small around an electron-hole pair, which is an electronically excited state. As a consequence, radiationless internal conversion (unimolecular decay converting electronic energy into heat) is extremely slow. Excited states decay radiatively in a defect-free, direct gap semiconductor such as CdSe. These simple spectroscopic facts have important practical consequences. Semiconductor lightemitting diodes have narrow emission bands and can show near $30 \%$ efficiency in converting electrical power into light. Semiconductor lasers and diodes also show excellent long-term stability against photochemical and current-induced degradation, when compared with many organic materials. All these properties reflect the strong chemical bonding, and the extremely delocalized nature of the electronic wave functions.

Semiconductor nanocrystals lie between the traditional regimes of chemistry and solid-state physics. ${ }^{1}$ Nanocrystal research was initially motivated by an effort to understand

Manoj Nirmal received his B.S in chemistry from the University of Chicago in 1990 followed by a Ph.D. from M IT in 1996 under Professor M. G. Bawendi. At MIT his thesis focused on the mechanism of radiative recombination in CdSe nanocrystals. As a recipient of an AT\&T Bell Laboratories Ph.D. scholarship he spent a year at Bell Labs conducting spectroscopy on single CdSe nanocrystals with Dr. J . Trautman, J . Macklin, T. Harris, and L. Brus. He was a postdoctoral student with Professor L. Brus at Columbia University between 1996 and 1998 and is currently a Senior Research Chemist at the Science Research Laboratory at $3 \mathrm{M}$.

Louis B rus has an undergraduate degree from Rice University and a Ph.D. from Columbia University, both in chemical physics. His graduate adviser was Richard Bersohn. As a Lieutenant in the U.S. Navy, he worked in the solid-state and chemistry divisions of the Naval Research Laboratory. In 1973 he joined the chemistry and materials research area of Bell Laboratories in Murray Hill, NJ. He joined the Columbia faculty in 1996. He has been interested in the structure and dynamics of transient chemical species, the flow of energy in molecules, local electromagnetic field effects, and nanocrystal chemistry and physics. He is presently chairman of the Board of Trustees of the Gordon Research Conferences. the evolution of bulk structural and electronic properties from the molecular scale. ${ }^{2}$ Presently, technological interest in nanocrystals stems from the prospect of creating novel materials with distinct physical properties. Nanocrystals act like molecules as they interact with light via their electronic transition dipoles. Yet, their delocalized solid-state parentage causes them to display unusual photophysics relative to molecules. In many molecules vibronic interaction in the excited state is strong as the wave function is localized on just one or a few bonds. The molecular excited state has a different structure which promotes fast nonradiative deactivation into the ground state. Emission quantum yields can be low, and often sensitive to quenching by the local environment. The situation is different in nanocrystals. In a $23 \AA$ diameter nanocrystal, for example, the wave function is delocalized over 100 unit cells with little probability density at the surface. This suggests that, in the absence of defects, internal or surface, a nanocrystal should exhibit near unity fluorescence quantum yield, and partial protection from quenching. The emission spectrum should be sharp as the Franck-Condon factors are small. At room temperature nanocrystals can be better photoemitters than bulk semiconductors because in nanocrystals the electron and hole remain superimposed due to quantum confinement. Nanocrystals have the potential to serve as ideal chromophores if their surface chemistry can be understood and controlled. In this Account we describe nanocrystal photophysics and make comparisons between inorganic solid-state materials and organic dye molecules.

\section{Intrinsic CdSe Quantum Mechanics}

The single electron molecular orbitals (MOs) in CdSe ( $E_{g}$ $\approx 1.7 \mathrm{eV}$ ) nanocrystals are formed from the HOMO and LUMO within each unit cell. Specifically, in CdSe the conduction, or lowest unoccupied, band is composed of $\mathrm{Cd} 5 \mathrm{~s}$ orbitals. The valence, or highest occupied, band formed from Se $4 p$ orbitals is more complex due to the 3-fold spatial degeneracy. While in organic molecules spin and orbital angular momentum are normally both good quantum numbers, in CdSe only $\mathrm{J}=\mathrm{L}+\mathrm{S}$ is a good quantum number due to strong spin-orbit coupling. The Sep HOMO yields $\mathrm{j}=3 / 2,1 / 2$ components. Furthermore, in the wurtzite structure the crystal field splits the $j=3 / 2$ component into $\mathrm{A}(\mathrm{j}= \pm 3 / 2)$ and $\mathrm{B}(\mathrm{j}= \pm 1 / 2)$ bands.

Quantum confinement occurs when the nanocrystal radius a becomes comparable to the bulk exciton Bohr radius ( $\sim 56 \AA$ for $\mathrm{CdSe}$ ). The simplest model for the electronic structure of CdSe nanocrystals is the particle in a sphere model in which the carriers are confined by a potential that becomes infinite at the interface. The nanocrystal has discrete instead of continuous MOs. Quantized values of electron wavelength translate into quantized energy levels with the energy of the first excited state of the electron or hole shifted by

$$
E_{1 s}=h^{2} / 8 m_{\text {eff }} a^{2}
$$


from its energy in the bulk material. The energy shifts, and therefore the nanocrystal band gap increases with decreasing size as $1 / a^{2}$. This strong size dependence is a practical advantage in use of nanocrystal luminescence. For example, CdSe nanocrystals of different sizes emit from 4500 to $6500 \AA$, across most of the visible spectrum. ${ }^{3}$

The synthesis of CdSe nanocrystals has improved considerably over the past decade. The current hightemperature organometallic synthesis ${ }^{4}$ yields highly monodisperse $(\sigma<5 \%)$, crystalline nanocrystals with radii tunable between 10 and $50 \AA$ having surfaces passivated with trioctylphosphine oxide (TOPO) and trioctylphosphine selenide (TOPSe) groups. These quantum dots exhibit "band edge", high quantum yield $\left(\phi_{\mathrm{f}} \approx 10 \%\right.$ at room temperature) emission. Luminescence from these high-quality samples reveals properties at variance with the simple particle in a sphere model. For example, transition dipole calculations based on particle in a sphere wave functions predicted nanosecond lifetimes, while microsecond lifetimes are observed.5,6 The band gap emission shows a short Franck-Condon progression in the LO vibration (the out of phase vibration of $\mathrm{Cd}$ and $\mathrm{Se}$ atoms) that is stronger than expected. ${ }^{6}$

To explain these results, an essentially qualitative surface model was first proposed.5,6 In this model, absorption of a photon results in the generation of an internal electron-hole pair. This is followed by fast localization of one of the carriers into a shallow surface trap. Hole localization was proposed to account for the reversal in luminescence polarization within $\sim 100 \mathrm{ps.} .^{5}$ The diminished overlap between the electron and hole due to localization would account for the long luminescence lifetime. Furthermore, the resulting electrical polarization within the nanocrystal would explain the coupling to the LO phonons. ${ }^{6}$

This model has not stood the test of time. The luminescence Stokes shift is insensitive to surface passivation. ${ }^{7}$ Furthermore, this picture cannot explain the strong magnetic field dependence of luminescence lifetimes. ${ }^{8}$ We understand now that these unexpected properties are quantitatively explained by electronic fine structure ${ }^{8,9,10}$ ignored in the simple model which, for example, does not include electron-hole exchange. $\mathrm{Ne}$ glecting exchange would be a fatal mistake in any small molecule theory; yet it is not unreasonable in a first nanocrystal model. Exchange splitting, for example, between excited singlets and triplets in organic molecules is on the order of $1 \mathrm{eV}$. However, in bulk semiconductors it is on the order of $10^{-4} \mathrm{eV}$ due to the extreme wave function delocalization. The electron-hole exchange Hamiltonian in CdSe is

$$
\hat{\mathrm{H}}_{\text {exch }}=-(2 / 3) \epsilon_{\text {exch }}\left(a_{0}{ }^{3}\right) \delta\left(r_{\mathrm{e}}-r_{\mathrm{h}}\right)(\hat{\sigma} \cdot \hat{\jmath})
$$

where $\hat{\sigma}(\hat{\jmath})$ is the electron (hole) spin $1 / 2(3 / 2)$ matrix, $a_{0}$ is the lattice constant, and $\epsilon_{\text {exch }}$ is the exchange strength constant. Since the exchange term is determined by the probability of finding the electron and hole at the same point, it will increase relative to the bulk (as the inverse

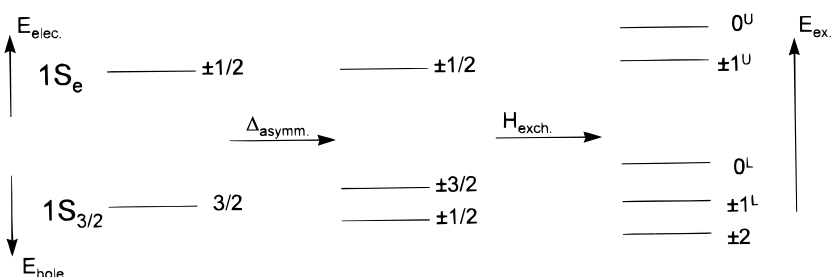

FIGURE 1. Illustration of the influence of the asymmetry and exchange perturbations on the 8-fold degenerate $1 S_{\mathrm{e}} 1 \mathrm{~S}_{3 / 2}$ electronhole pair state. $E_{\text {elec }}$ and $E_{\text {hole }}$ refer to the energies of the electron and the hole. The exchange term mixes the electron and hole angular momenta, yielding five states labeled by the exciton total angular momentum $\left(V_{m}+m_{z}\right)$. $E_{\text {ex }}$ is the total exciton energy.

volume) due to confinement of the electron and hole wave functions. The exchange interaction evolves toward the molecular regime.

The influence of the exchange term and nanocrystal asymmetry on the band edge electronic fine structure is indicated in Figure 1. For hypothetical spherical nanocrystals with the zinc blende lattice structure, the band edge state, $1 S_{e} 1 S_{3 / 2}$, with both the electron and hole in $1 S$ totally symmetric particle in a sphere type states, is 8-fold degenerate taking into account the electron spin and hole spatial degeneracy. In wurtzite nanocrystals $s^{4}$ the crystal field along with any asymmetry in the overall shape of the nanocrystal splits the $1 S_{3 / 2}$ hole state into $\mathrm{j}= \pm 3 / 2$, $\pm 1 / 2$ states. The $\hat{\sigma} \cdot \hat{\jmath}$ exchange term mixes the electron and hole angular momenta, yielding five excitonic states labeled by the exciton total angular momentum projection F. Both the lowest \pm 2 and $0^{L}$ states are optically passive within the electric dipole approximation. For band edge excitation the lowest optically active $\pm 1^{\mathrm{L}}$ state is predominantly populated. This is followed by radiationless relaxation to the \pm 2 dark excitonic state. Emission from this weakly emitting state accounts for the long microsecond luminescence lifetime. A small Stokes shift of the band edge excited luminescence is caused by the size dependent exchange splitting between the \pm 2 and $\pm 1^{\mathrm{L}}$ states. ${ }^{8}$ The magnetic field dependence of the luminescence lifetimes and LO phonon couplings (Figure 2) are also explained by this model. ${ }^{8}$ The microsecond decay seen at zero field decreases with increasing magnetic field. Also shown is the field dependence of the vibronic spectra. In isolation the \pm 2 state would have an infinite lifetime since the photon cannot carry an angular momentum of 2 within the electric dipole approximation. However the \pm 2 state can recombine via an LO phonon-assisted momentum-conserving transition. Consequently at zero field, the zero phonon line is strongly suppressed while higher LO phonon replicas are enhanced. With increasing magnetic field the zero phonon line intensity increases dramatically. With increasing field the \pm 2 state gains oscillator strength from higher lying optically active states. This explains the decrease in luminescence lifetime with increasing field. Also since the \pm 2 state gains optically active character, this diminishes the need for LO phononassisted recombination which explains the increase in zero phonon line emission with field. The apparent coupling to LO phonons, determined from the vibronic spectrum, 

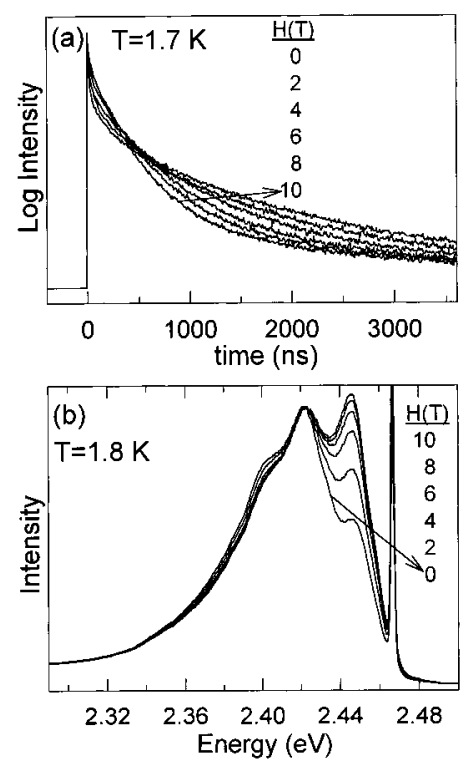

FIGURE 2. Magnetic field dependence of (a) emission decays recorded at the peak of the luminescence $(2.436 \mathrm{eV})$ with a pump energy of $2.736 \mathrm{eV}$ and (b) fluorescence line-narrowed spectra excited at the band edge (2.467 eV) for $12 \AA$ radius nanocrystals and normalized to their one LO phonon line. A small fraction of the excitation laser is included to mark the pump position. Experiments were carried out in the Faraday geometry with the field $\mathbf{H}$ parallel to $\mathbf{k}$, the light wave vector.

is a consequence of the fact that at low temperatures, where repopulation of the $\pm 1^{\mathrm{L}}$ state is negligible, LO phonons must participate in momentum-conserving transitions for emission. In reality however coupling to LO phonons is weak as evidenced by the long microsecond luminescence lifetime.

While the exchange interaction in CdSe nanocrystals is enhanced by up to 2 orders of magnitude, relative to the bulk, exchange splittings remain small compared to kT at room temperature. "Dark" excitonic effects are thus not observed at room temperature. In organic molecules, however, where singlet-triplet splittings could be on the order of $\sim 1 \mathrm{eV}$, intersystem crossing into the triplet state can strongly influence luminescence count rates even at room temperature.

\section{Surface Passivation and Shell Nanocrystals}

While the "band edge" emission is intrinsic in origin, the surface still provides sites for nonradiative recombination. As synthesized, the TOPO/TOPSe passivated nanocrystals exhibit room-temperature quantum yields between 5 and $10 \%$. Substitution of the TOPO/TOPSe surface capping groups results in variations in the luminescence yield and a suppression/increase in the "deep trap" emission. ${ }^{7}$ However, the energetics of the band edge emission appear unaltered, demonstrating the intrinsic origin of this component. To increase the quantum yield requires the elimination of midgap surface states with appropriate surface passivation.

Even as early as 1982 it was observed that adsorption of $\mathrm{Zn}$ ions onto $\mathrm{CdS}$ particles increased luminescence quantum yields, and this effect was understood in terms of a higher band gap material (ZnS) passivating a lower band gap material (CdS). ${ }^{11}$ Modern high-temperature organometallic synthesis can grow a near epitaxial monolayer of ZnS on CdSe nanocrystals. 3,12 These shell nanocrystals exhibit quantum yields of up to $50 \%$ at room temperature, a clear improvement over the organically terminated nanocrystals discussed above. However, the $12 \%$ difference in bond lengths between CdSe and ZnS results in significant lattice mismatch and increasing strain with ZnSthickness. Recently CdS-overcoated CdSe nanocrystals have also been synthesized. ${ }^{13}$ CdS appears to be an ideal shell material for CdSe. The lattice mismatch of $3.9 \%$ permits epitaxial growth of the CdS shell. An added advantage results from differences in confinement of the electron and hole in this system. Unlike ZnS-overcoated particles, while the hole is confined to within the CdSe core, the electron is delocalized over the entire nanocrystal. This permits electrical access to the nanocrystal which is critical in devices such as photovoltaic cells requiring electron conduction. Encapsulated nanocrystals also exhibit improved photostability. Single nanocrystal luminescence measurements (following section) indicate that the "best" nanocrystals emit $\gg 10^{8}$ photons before permanently photobleaching. This represents a significant advantage over organic chromophores.

\section{Single Nanocrystal Luminescence}

The strong size dependence of nanocrystal optical properties, while an asset in their practical use, ${ }^{14}$ represents a problem in scientific experiments. Even the best samples have distributions of size, shape, and surface chemistry, and thus have varying optical properties. Ideally the true single nanocrystal properties can be obtained by hole burning and size selective luminescence experiments. ${ }^{15}$ Yet, despite a decade of such work, recent single nanocrystal luminescence studies have revealed new processes in nanocrystals, such as spectral diffusion and the random intermittency of the fluorescence. ${ }^{16,17}$ Low-temperature single nanocrystal measurements are discussed in this issue by Empedocles and Bawendi.

Figure $3 a$ is a fluorescence image of single CdSe nanocrystals embedded in a thin polymer film. The streaks in the image arise due to the discrete turning on/ off of single nanocrystal emission while acquiring this raster scanned image. This is illustrated in a fluorescence intensity versus time trace of a single CdSe nanocrystal (Figure 3b). Remarkably the luminescence turns on/off ("blinks") on a several second time scale. In previous ensemble experiments blinking was missed, as it averages to zero when many nanocrystals are observed simultaneously. Fluorescence intensity versus time traces taken at low and at high intensity (Figure 4a) show that the "on" period duration scales inversely with excitation intensity, while the "off" period appears to be intensity independent. This result suggests that the nonemissive state is created via the nanocrystal excited state. The bright state is however recreated by a spontaneous thermal process from the nonemissive state. The fluorescence time trace of a 
a)

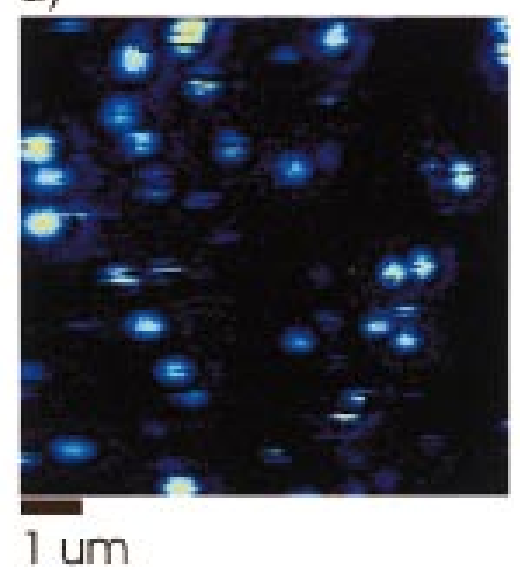

b)

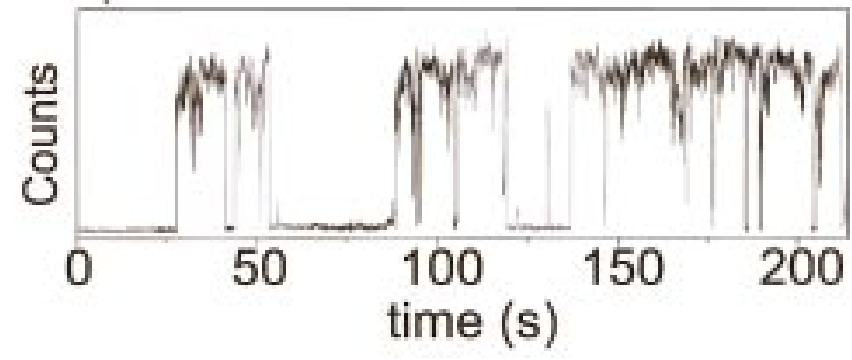

FIGURE 3. (a) Image of a random field of single $21 \AA$ radius $C d S e$ nanocrystals with $\sim 4$ monolayers of $Z n S$ on the surface, acquired by raster scanning the sample across a diffraction-limited laser spot $(\lambda=532 \mathrm{~nm}$, fwhm $\approx 0.38 \mu \mathrm{m})$ and collecting the red-shifted fluorescence onto an avalanche photodiode in an epi-illumination confocal geometry. (b) Fluorescence intensity versus time trace of a single $21 \AA$ radius CdSe nanocrystal with a $40 \mathrm{~ms}$ sampling interval and an excitation intensity of $\sim 0.52 \mathrm{~kW} / \mathrm{cm}^{2}$.

TOPO/TOPSe organically capped nanocrystal is compared in Figure $4 \mathrm{~b}$ with that of a ZnS-overcoated one having seven monolayers of ZnS on the surface. Both the average on and off times increase dramatically in the ZnSovercoated nanocrystal. To confirm this trend, we measured average on/off times as a function of ZnS shell thickness from "bare" nanocrystals to those with $0.25,2$, and 7 monolayers of $\mathrm{ZnS}$ on the surface. Both the average on and off times increase with increasing ZnS shell thickness.

One mechanism consistent with the on/off behavior involves Auger nonradiative photoionization. ${ }^{16,18,19}$ At our excitation intensities $\left(\sim 0.5 \mathrm{~kW} / \mathrm{cm}^{2}\right)$ we typically excite each nanocrystal every $10^{-5} \mathrm{~s}$, and the nanocrystals decay in about $10^{-8} \mathrm{~s}$. Only very rarely can two electron-hole pairs be simultaneously excited. However, if there are two pairs, then photoionization should be fast. In such nanocrystals the ca. $2 \mathrm{eV}$ energy released from the annihilation of one electron-hole pair may be transferred to the remaining carriers, one of which can then be ejected into the matrix. If the resulting ionized nanocrystal is subsequently photoexcited, then Auger nonradiative decay, a purely electronic process unrelated to FranckCondon factors, creates a very low emission quantum yield. ${ }^{19}$ In this Auger process, the photogenerated elec-
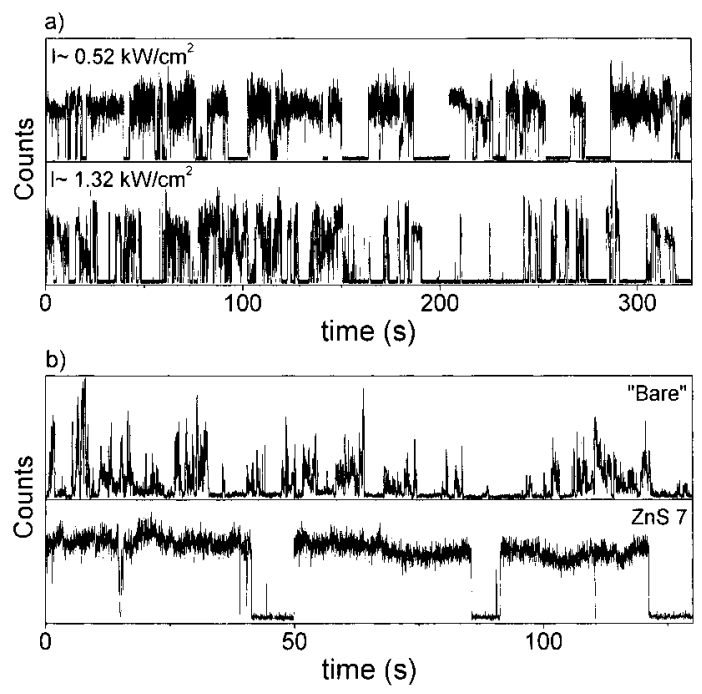

FIGURE 4. (a) Comparison of fluorescence intensity versus time traces at $\sim 0.52 \mathrm{~kW} / \mathrm{cm}^{2}$ and at $\sim 1.32 \mathrm{~kW} / \mathrm{cm}^{2}$ with a sampling interval of $10 \mathrm{~ms}$. (b) Fluorescence intensity versus time traces of a "bare", TOPO/TOPSe-passivated nanocrystal compared with that of a ZnS-overcoated one with a shell thickness of $\sim 7$ monolayers at the same excitation intensity $\left(I \approx 0.70 \mathrm{~kW} / \mathrm{cm}^{2}\right)$ and a sampling interval of $20 \mathrm{~ms}$.

tron-hole pair recombines by transferring its energy to the strongly coupled, resident third carrier; this is why an ionized nanocrystal is nonemissive. Eventually the nanocrystal is neutralized via a second photoionization event or the return of the ejected carrier. Neutralization restores the emission. Since the on time is determined by the ease of ionization across the interface, and the off period by the time it takes for the ejected carrier to tunnel back through the same interface, ZnS-overcoated nanocrystals exhibit longer on/ off times as expected from this model.

This simple proposal must be tested by further experiment. Nevertheless, single nanocrystal measurements have revealed new aspects of the photophysics obscured in previous ensemble measurements. Although photoionization is rare (probability/ excitation $\sim 10^{-6}$ ), it has a significant effect on luminescence count rates and quantum yields due to the unusually long $\left(\tau_{\mathrm{n}} \approx 0.5 \mathrm{~s}\right)$ neutralization times during which an Auger nonradiative recombination mechanism dominates. Ensemble measurements alone would have led us to attribute the increase in quantum yield in $\mathrm{ZnS}$-overcoated particles to a decrease in some fast unimolecular nonradiative processes on the same $10^{-8} \mathrm{~s}$ time scale as the luminescence.

\section{Silicon Nanocrystals}

With features in Si computer chips approaching $10 \mathrm{~nm}$, there is a pressing need to understand the electronic and transport properties of indirect gap silicon nanocrystals. How does the indirect nature of the band gap in bulk silicon develop with size? The band gap optical transition is dipole forbidden in the infinite crystal, where translational symmetry is exact. In nanocrystals, however, theory indicates the transition will be weakly dipole allowed.20

Unlike bulk Si, Si nanocrystals photoluminesce with high quantum yield. This first became apparent in porous 

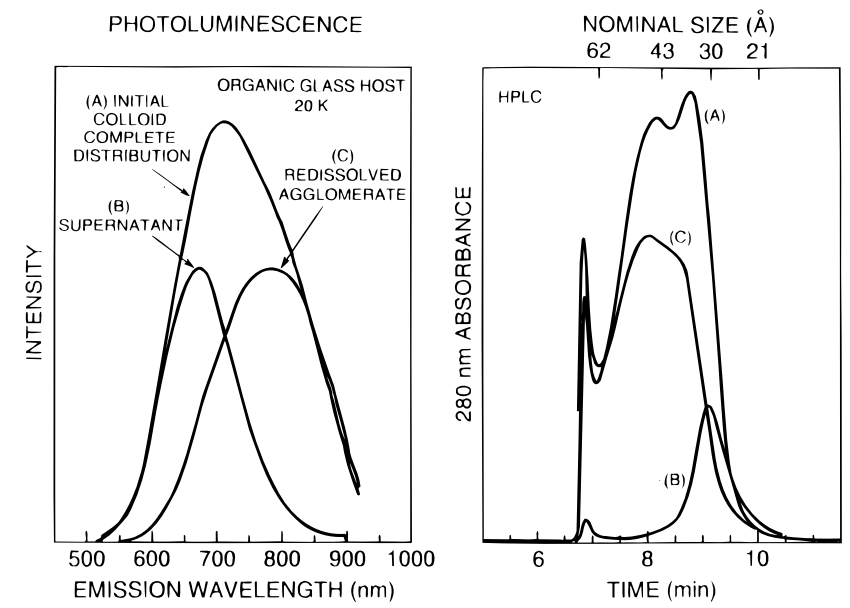

FIGURE 5. (A) Spectrally corrected luminescence spectra (350 nm excitation) in organic glass (20 K). Traces: 1, initial colloid; 2 , supernatant; 3, redissolved agglomerate. (B) Corresponding HPLC size distributions. The spike at $6.7 \mathrm{~min}$ is the column large size exclusion limit and does not indic ate a specific size. The logarithmic size caibration is approximate. Trace numbers as in (A). Adapted from ref 24.

$\mathrm{Si}$ (por-Si) thin films, made by electrochemical etching of $\mathrm{Si}$ wafers in HF solution. ${ }^{21}$ This complex material is composed of partially fused crystalline nanocrystals and nanowires; it can be as much as $80 \%$ porous. Rigorous EXAFS measurements on $\mathrm{H}$-passivated pores reveal a mean silicon coordination number of $<3$, indicating that a significant fraction of the atoms are on the surface, bonded to hydrogen. ${ }^{22}$

Isolated silicon nanocrystals with about a $1 \mathrm{~nm}$ thick $\mathrm{SiO}_{2}$ surface passivating layer can be synthesized by a high-temperature aerosol reaction. ${ }^{23}$ These Si nanocrystals and por-Si show very similar broad Gaussian luminescence spectra between 550 and $900 \mathrm{~nm}$, and similar long lifetimes. ${ }^{21,24,25}$ Both $\mathrm{H}$-terminated and oxideterminated por-Si display the same emission spectra as the oxide-terminated nanocrystals. Thus, the chemical nature of the passivation does not directly influence luminescence. The nanocrystal synthesis yields a fairly wide distribution of particle diameters. A narrowing of the distribution can be achieved by size exclusion chromatography or by size selective precipitation, in which the addition of a nonsolvent selectively precipitates the largest particles within the distribution (Figure 5). ${ }^{24,25}$

The oxide shell can be the ideal passivation for silicon nanocrystals, driving all midgap surface states far out of the band gap. The importance of the surface in nonradiative processes is evidenced by the fact that the quantum yield of the as-synthesized nanocrystals can be significantly improved by refluxing them in acidic $\mathrm{H}_{2} \mathrm{O}_{2}$. This procedure presumably oxidizes any residual surface silicon dangling bonds at the $\mathrm{Si} / \mathrm{SiO}_{2}$ interface. These ideas and methods are borrowed from the passivation of $\mathrm{Si} / \mathrm{SiO}_{2}$ interfaces in microelectronic devices.

Si nanocrystals can be optically characterized as dilute colloids in ethylene glycol. The smallest, 1-2 nm diameter Si nanocrystals luminesce at $\sim 2.0 \mathrm{eV}$, which represents a blue shift of $\sim 0.9 \mathrm{eV}$ relative to the bulk band gap of $1.1 \mathrm{eV}$. UV excitation spectra, measurement of emission lifetime, direct measurement of absolute quantum yield, and size-selective luminescence at low temperature all indicate that $\mathrm{Si}$ nanocrystals remain indirect gap, with $10^{-5}-10^{-3}$ s radiative lifetimes, down to the smallest sizes observed. Acidic $\mathrm{H}_{2} \mathrm{O}_{2}$ "activated" nanocrystals exhibit a room-temperature quantum yield of $\sim 5 \%$, which increases to $\sim 50 \%$ below $50 \mathrm{~K}$. The relative contributions of the radiative $\left(\gamma_{\mathrm{r}}\right)$ and nonradiative $\left(\gamma_{\mathrm{nr}}\right)$ rates to the measured lifetimes can be determined by determining the absolute quantum yield as a function of temperature. Below $50 \mathrm{~K}$ the quantum yield stays constant, while the lifetime continues to increase. This suggests that at sufficiently low temperatures $\gamma_{\mathrm{r}} \gg \gamma_{\mathrm{nr}}$ and the 50\% quantum yield reflects two populations of particles: $50 \%$ of the particles luminesce with a quantum yield of 1 while the other half are nonfluorescent due to the presence of a catastrophic defect.

The strong similarities between silicon nanocrystals and por-Si lead to some general comparisons between radiative and nonradiative processes in low-dimensional silicon versus the bulk. This analysis points again to the critical importance of slow internal conversion in bulk semiconductors. At liquid helium temperatures an excitonic electron-hole $(\mathrm{e}-\mathrm{h})$ pair is bound because the dissociation energy (14.7 meV) 26 is larger than $\mathrm{kT}$. Such a bound exciton has dimensions $(\sim 3 \mathrm{~nm})$ similar to those of a silicon nanocrystal. ${ }^{27}$ Both systems (bulk trapped exciton and passivated nanocrystal) lack translational symmetry, but experimentally remain indirect gap. Both experimentally have a luminescence quantum yield of essentially 1 in the absence of defects. Internal conversion across the band gap is extremely slow, even on the $10^{-3} \mathrm{~s}$ time scale.

At room temperature, however, the bulk and nanoscale photophysics are quite different. When $\mathrm{kT}$ is greater than the exciton binding energy in the bulk, the e-h pairs dissociate, and the individual carriers travel macroscopic distances. Carriers can interact and recombine nonradiatively via a three-body, purely electronic Auger process: $(e+h)+(e$ or $h) \rightarrow(e$ or $h)+1.1 \mathrm{eV}$. This is the same process previously suggested as the reason ionized CdSe nanocrystals are nonluminescent. Due to the relatively high mobilities and long lifetimes of carriers in high-quality crystalline $\mathrm{Si}$, Auger processes dominate even at moderate intensities. A second nonradiative process involves deactivation at defects and traps: even at densities as low as $10^{14} / \mathrm{cm}^{3}$, traps can dominate the bulk photophysics for long lifetimes.

In passivated and isolated nanocrystals, however, both processes are diminished at room temperature. ${ }^{25}$ Even at moderate excitation intensities it is unlikely to create two excitations within a nanocrystal since the optical absorption cross section roughly scales as the particle volume. Furthermore, since the mobility of photogenerated carriers is limited by the nanocrystal dimensions, defects in one nanocrystal cannot influence radiative processes in others. This leads to the conclusion that the quantum yield in $\mathrm{Si}$ nanocrystals and por-Si is high due 
to a reduction of trapping and Auger processes. In any electronic material with low mobility, geminate carrier recombination is enhanced.

\section{Electron Transport in Nanocrystal Materials}

The small lattice distortion occurring around carriers creates the high mobility that enables semiconductor devices to operate at high frequency. A remarkable further consequence is the fact that electron transport in materials incorporating touching nanocrystals is sensitive to environmental control.

The optical and electrical properties of $80 \%$ porous $\mathrm{Si}$ films, epitaxially etched on Si wafers, can be studied in either "wet" or "dry" form. The pores can be evacuated in a vacuum, or filled with liquid in essentially an electrochemical liquid junction configuration. It was discovered quite early in the exploration of por-Si properties that both optical and electrical properties were very sensitive to minute amounts of polar materials into the pore structure. $25,29,30$ For example, dry porous silicon emits strongly as we have described, but introduction of just a monolayer of methanol on the pore surfaces leads to dynamic quenching. This effect is reversible if the methanol is pumped away. In electrical properties, the introduction of methanol can reversibly raise the conductivity by orders of magnitude.

To a physical chemist, these effects are reminiscent of kinetic solvation effects in the Marcus-Hush model for electron transfer in proteins and organic molecules. ${ }^{31}$ Consider the model problem of electron transfer between two touching Si nanocrystals. ${ }^{32}$ The transfer free energy is approximated by the difference in nanocrystal electron affinities. Bulk Si has a near $4 \mathrm{eV}$ electron affinity; this is the position of the conduction band edge with respect to an electron at rest in a vacuum. In a Si nanocrystal the electron affinity is smaller due to two effects: size quantization as discussed before and the loss of dielectric solvation energy. The difference in nanocrystal electron affinity can be large; for example, the free energy exothermicity for transfer from a $2 \mathrm{~nm}$ to a $4 \mathrm{~nm}$ nanocrystal is about $0.5 \mathrm{eV}$ in a vacuum.

The inner reorganization energy for transfer between Si nanocrystals is very small, on the order of $10^{-2} \mathrm{eV}$. This is simply a restatement of the fact that there is little lattice distortion around a free electron. This electron's electric field exits the nanocrystal and interacts with the local environment; this is the source of the outer reorganization energy in Marcus-Hush theory. If the pores are evacuated, then the total reorganization energy is small. For the $2 \mathrm{~nm}$ to $4 \mathrm{~nm}$ transfer case, the rate will lie in the Marcus inverted region and be very slow, unless there is an accidental electronic degeneracy between the donor energy and an excited level in the acceptor. However, if the outer reorganization energy is large because of polar molecules in the pores, then exothermic electron transfer can occur rapidly (Figure 6). In porous Si, internally adsorbed polar molecules facilitate electron transfer among nanocrystals of differing size; they serve to enable hopping

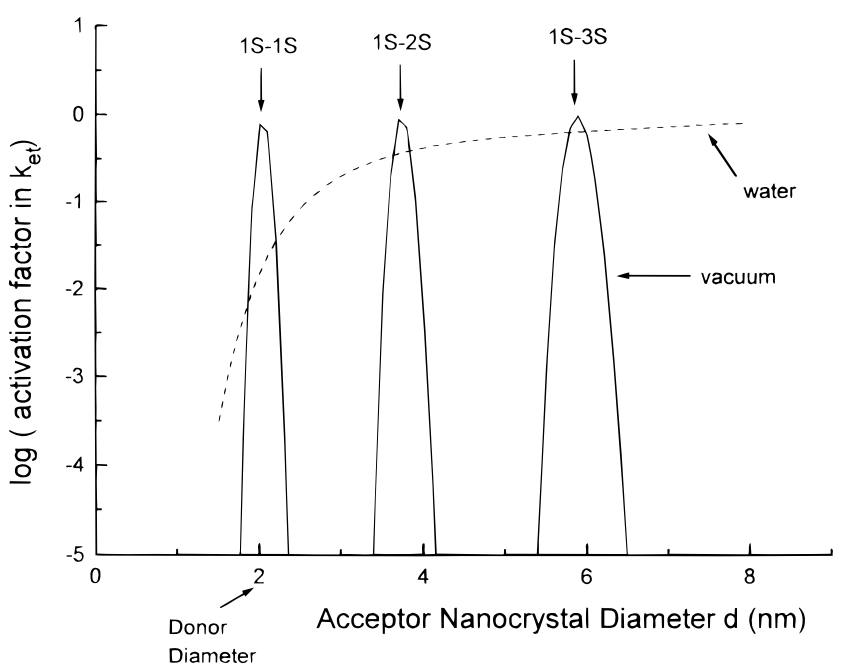

FIGURE 6. Logarithmic plot (base 10) of high-temperature Marcus activation energy, for electron transfer from a $2 \mathrm{~nm}$ donor $\mathrm{Si}$ nanocrystal to acceptor nanocrystals of varying size. $1 S-2 S$ refers to $1 S$ inital donor state and $2 S$ final acceptor state. Solid line refers to transfer in a vacuum, and the dashed line to transfer in water. Adapted from ref 32 .

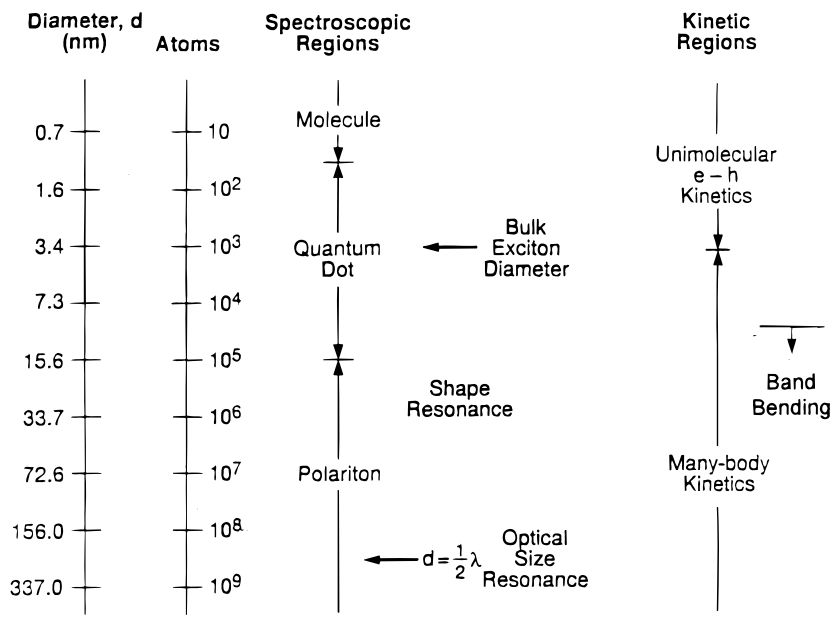

FIGURE 7. Schematic representation of physical size regimes for spectroscopic and excited-state photophysical nanocrystal properties, adapted from ref 25.

electron mobility under an applied field. Also in photoluminescence, polar molecules serve to enable the trapping of carriers on midgap surface electronic states. This trapping competes with photoluminescence.

\section{Final Comments}

Many groups have contributed to the partial understanding of nanocrystal internal quantum mechanics which has been achieved over the past 15 years. An outline of various physical size regimes is shown in Figure 7.25 We have seen in both $\mathrm{Si}$ and CdSe that the luminescence quantum yield is high if the surface chemistry can be controlled on an otherwise defect free nanocrystal. Intrinsic radiationless decay across the band gap is slow. Yet in both systems the surface chemistry and trapping dynamics, while understood in outline form, are not yet sufficiently controlled to make these nanocrystals into 
ideal chromophores. Even passivated nanocrystals do have surfaces, and perhaps any nanostructured material with surfaces will never be as stable as a bulk inorganic semiconductor under prolonged current flow or optical irradiation. Yet note that the $\mathrm{SiO}_{2} / \mathrm{Si}$ planar interface, so important in computer technology, has been nearly perfected by a combination of hydrogen and oxygen reactive chemistry. This result encourages further research to achieve better, perhaps "ideal" chromophores.

Note Added in Proof. High quantum yield Si nanocrystal luminescence has recently been proposed as the source of extended red emission in the interstellar medium. 33,34 Also, surface-functionalized CdSe nanocrystals have been used as luminescent probes in biological imaging. In some cases they are more stable against photobleaching than functionalized organic dyes. ${ }^{35,36}$

\section{References}

(1) Brus, L. E. Quantum Crystallites and Nonlinear Optics. Appl. Phys. A 1991, 53, 465-474. Alivisatos, A. P. Semiconductor clusters, nanocrystals, and quantum dots. Science 1996, 271, 933-937.

(2) Rossetti, R.; Nakahara, S.; Brus, L. E. Quantum size effects in the redox potentials, resonance Raman spectra, and electronic spectra of CdS crystallites in aqueous solution. J. Chem. Phys. 1983, 79, 10861088.

(3) Dabbousi, B. O.; Rodrigo-Viejo, J.; Mikulec, F. V.; Heine, J. R.; Mattousi, H.; Ober, R.; Jensen, K. F.; Bawendi, M. G. (CdSe)ZnS Core Shell Quantum Dots: Synthesis and Characterization of a Size Series of Highly Luminescent Nanocrystallites. J. Phys. Chem. 1997, 101, 9463-9475.

(4) Murray, C. B.; Norris, D. J.; Bawendi, M. G. Synthesis and Characterization of Nearly Monodisperse CdE $(\mathrm{E}=\mathrm{S}, \mathrm{Se}, \mathrm{Te}$ ) Semiconductor Nanocrystallites. J. Am. Chem. Soc. 1993, 115, 8706-8715. Bowen Katari, J. E.; Colvin, V. L.; Alivisatos, A. P. X-ray Photoelectron Spectroscopy of CdSe Nanocrystals with Applications to Studies of the Nanocrystal Surface. J. Phys. Chem. 1994, 98, 4109-4117.

(5) Bawendi, M. G.; Carroll, P. J.; Wilson, W. L.; Brus, L. E. Luminescence Properties of CdSe Quantum Crystallites - Resonance between Interior and Surface Localized States. J. Chem. Phys. 1992, 96, 946-954.

(6) Nirmal, M.; Murray, C. B.; Bawendi, M. G. Fluorescence Line Narrowing in CdSe Quantum Dots Surface Localization of the Photogenerated Exciton. Phys. Rev. B 1994, 50, 2293-2300.

(7) Kuno, M.; Lee, J. K.; Babbousi, B. O.; Mikulec, F. V.; Bawendi, M. G. The band edge luminescence of surface modified CdSe nanocrystallites: Probing the luminescing state. J. Chem. Phys. 1997, 106, 98699882.

(8) Nirmal, M.; Norris, D. J.; Kuno, M.; Bawendi, M. G.; Efros, Al. L.; Rosen, M. Observation of the DarkExciton in CdSe Quantum Dots. Phys. Rev. Lett. 1995, 75, 3728-3731.

(9) Norris, M. G.; Bawendi, M. G. Structure in the Lowest Absorption Feature of CdSe Quantum Dots. J. Chem. Phys. 1995, 103, 5260-5268.

(10) Efros, Al. L.; Rosen, M.; Kuno, M.; Nirmal, M.; Norris, D. J.; Bawendi, M. G. Band-edge exciton in quantum dots of semiconductirs with a degenerate valence band: Dark and Bright exciton states. Phys. Rev. B 1996, 54, 4843-4856.
(11) Rossetti, R.; Brus, L. Electron-Hole Recombination Emission as a Probe of Surface Chemistry in Aqueous CdS Colloids. J. Phys. Chem. 1982, 86, 44704472.

(12) Hines, M. A.; Guyot-Sionnest, P. Synthesis and Characterization of Strongly Luminescing ZnSCapped CdSe Nanocrystals. J. Phys. Chem. 1996, $100,468-471$.

(13) Peng, X.; Schlamp, M. C.; Kadavanich, A. V.; Alivisatos, A. P. Epitaxial Growth of Highly Luminescent CdSe/CdS Core/Shell nanocrystals with Photostability and Electronic Accessibility J. Am. Chem. Soc. 1997, 119, 7019-7029.

(14) Colvin, V. L.; Schlamp, M. C.; Alivisatos, A. P. Light Emitting Diodes made from Cadmium Selenide Nanocrystals and a Semiconducting Polymer. Nature 1994, 370, 354-357. Dabbousi, B. O.; Bawendi, M. G.; Onitsuka, O.; Rubner, M. F. Electroluminescence from CdSe Quantum-Dot Polymer Composites. Appl. Phys. Lett. 1995, 66, 1316-1318.

(15) (a) Alivisatos, A. P.; Harris, A. L.; Levinos, N. J.; Steigerwald, M. L.; Brus, L. E. Electronic States of Semiconductor Clusters - Homogeneous and Inhomogeneous Broadening of the Optical Spectrum. J. Chem. Phys. 1988, 89, 4001-4011. (b) Bawendi, M. G.; Wilson, W. L.; Rothberg. L.; Carroll, P. J.; Jedju. T. M.; Steigerwald, M. L.; Brus. L. E. Electronic Structure and Photoexcited Carrier Dynamics in Nanometer Size CdSe Clusters. Phys. Rev. Lett. 1990, 65, 1623-1626.

(16) Nirmal, M.; Dabbousi, B. O.; Bawendi, M. G.; Macklin, J. J.; Trautman, J. K.; Harris, T. D.; Brus, L. E. Fluorescence intermittency in single cadmium selenide nanocrystals. Nature 1996, 383, 802-804.

(17) Empedocles, S. A.; Norris, D. J.; Bawendi, M. G. Photoluminescence Spectroscopy of Single CdSe Nanocrystallite Quantum Dots. Phys. Rev. Lett. 1996, 77, 3873-3876. Empedocles, S. A.; Bawendi, M. G. Quantum-Confined Stark Effect in Single CdSe Nanocrystallite Quantum Dots. Science 1997, 278, 2114-2117.

(18) Efros, Al. L.; Rosen, M. Random telegraph signal in the photoluminescence intensity of a single quantum dot. Phys. Rev. Lett. 1997, 78, 1110-1113.

(19) Chepic, D. I,; Efros, Al. L.; Ekimov, A. L.; Ivanov, M. G.; Kharchenko, V. A.; Kudriavtsev, I. A.; Yazeva, T. V. Auger Ionization of Semiconductor Quantum Drops in a Glass Matrix. J. Lumin. 1990, 47, 113127.

(20) Takagahara, T.; Takeda, K. Theory of the quantum confinement effect on excitons in quantum dots of indirect-gap materials. Phys. Rev. B 1992, 46, 1557815581.

(21) Canham, L. T. Silicon Quantum Wire Array Fabrication by Electrochemical and Chemical Dissolution of Wafers. Appl. Phys. Lett. 1990, 57, 1046-1048. Koyama, H.; Araki, M.; Yamamoto, Y.; Koshida, N. Visible Photoluminescence of Porous $\mathrm{Si}$ and its Related Optical Properties. Jpn. J. Appl. Phys. 1991, 30, 3606-3609. Petrova-Koch, V.; Muschik, T.; Kux, A.; Meyer, B. K.; Koch, F.; Lehmann, V. Rapid Thermal Oxidized Porous $\mathrm{Si}$ - The Superior Photoluminescent Si. Appl. Phys. Lett. 1992, 61(8), 943945.

(22) Schuppler, S.; Friedman, S. L.; Marcus, M. A.; Adler, D. L.; Xie, Y. H.; Ross, F. M.; Harris, T. D.; Brown, W. L.; Chabal, Y. J.; Brus, L. E.; Citrin, P. H. Dimensions of Luminescent Oxidized and Porous Silicon Structures. Phys. Rev. Lett. 1994, 72, 26482651. 
(23) Littau, K. A.; Szajowski, P. J.; Muller, A. J.; Kortan, A. R.; Brus, L. E. A Luminescent Silicon Nanocrystal Colloid via a High-Temperature Aerosol Reaction. J. Phys. Chem. 1993, 97, 1224-1230.

(24) Wilson, W. L.; Szajowski, P. F.; Brus, L. E. Quantum Confinement in Size-Selected, Surface-Oxidized Silicon Nanocrystals. Science 1993, 262, 1242-1244.

(25) Brus, L. E.; Szajowski, P. F.; Wilson, W. L.; Harris, T. D.; Schuppler, S.; Citrin, P. H. Electronic Spectroscopy and Photophysics of Silicon Nanocrystals: Relationship to Bulk c-Si and Porous Si. J. Am. Chem. Soc. 1995, 117, 2915-2922.

(26) Shaklee, K. L.; Nahory, R. E. Valley-Orbit Splitting of Free Excitons? The Absorption Edge of Si. Phys. Rev. Lett. 1970, 24, 942-945.

(27) Haynes, J. R.; Lax, M.; Flood, W. F. Proc. Int. Conf. Semicond. Phys. 1961, 423. Cuthbert. J. D. Phys. Rev. 197, B1, 1552.

(28) Ben-Chorin, M.; Kux, A.; Schechter, I. Adsorbate Effects on Photoluminescence and Electrical Conductivity of Porous Silicon. Appl. Phys. Lett. 1994, 64, 481-483.

(29) Li, K.; Tsai, C.; Sarathy, J.; Campbell, J. ChemicallyInduced Shifts in the Photoluminescence Spectra of Porous Silicon. Appl. Phys. Lett. 1993, 62, 31923194. Dubin, V. M.; Ozanam, F.; Chazalviel, J. Electronic States of Photocarriers in Porous Silicon Studies by Photomodulated Infrared Spectroscopy. Phys. Rev. B 1994, 50, 14867-14880. Ichinole, T.; Nozaki, S.; Ono, H.; Morisaki, H. Cyclic shifts in the photoluminescnce spectra of the porous $\mathrm{Si}$ in HF. Appl. Phys. Lett. 1995, 66, 1644-1646.

(30) Halimaoui, A. et al. Electroluminescence in the Visible Range During Anodic-Oxidation of Porous Silicon Films. Appl. Phys. Lett. 1991, 59, 304-306.
Bressers, P.; Knapen, J.; Meulenkamp, E.; Kelly, J. Visible light emission from a porous silicon diode. Ibid. 1992, 61, 108-110. Bsiesy, A.; et al. VoltageControlled Spectral Shift of Porous Silicon Electroluminescence. Phys. Rev. Lett. 1993, 71, 637-640. Ligeon, M.; et al. Analysis of the Electroluminescence Observed During the Anodic Oxidation of Porous Layers Formed on Lightly p-Doped Silicon. J. Appl. Phys. 1993, 74, 1265-1271. Kooij, E.; Despo, R.; Kelly, J. Electroluminescence from porous silicon due to electron injection from solution. Appl. Phys. Lett. 1995, 66, 2552-2554.

(31) Marcus, R. A. Chemical and Electrochemical ElectronTransfer Theory. Annu. Rev. Phys. Chem. 1964, 15, 155-196.

(32) Brus. L. Model for carrier dynamics and photoluminsecence quenching in wet and dry porous Si thin films. Phys. Rev. B 1996, 53, 4649-4656.

(33) Witt, A.; Gordon, K.; Furton, D. Silicon Nanoparticles: Source of Extended Red Emission? Astrophys. J. 1998, 501, L111-L115.

(34) Ledoux, G.; et al. Silicon as a Candidate Carrier for ERE. Astron. Astrophys. 1998, 333, L39-L43.

(35) Bruchez, M.; Moronne, M.; Gin, P.; Weiss, S.; Alivisatos, A. P. Semiconductor Nanocrystals as Fluorescent Biological Labels. Science 1998, 281, 20132016.

(36) Chan, W. C. W.; Nie, S. Quantum Dot Bioconjugates for Ultrasensitive Nonisotopic Detection. Science 1998, 281, 2016-2018.

AR9700320 